\title{
Modeling User Quality of Experience (QoE) through Position Discrepancy in Multi-Sensorial, Immersive, Collaborative Environments
}

\author{
Shanthi Vellingiri \\ University of Texas at Dallas \\ Richardson, Texas \\ USA \\ shanthi.vellingiri@utdallas.edu
}

\author{
Prabhakaran Balakrishnan \\ University of Texas at Dallas \\ Richardson, Texas \\ USA \\ praba@utdallas.edu
}

\begin{abstract}
Users' QoE (Quality of Experience) in Multi-sensorial, Immersive, Collaborative Environments (MICE) applications is mostly measured by psychometric studies. These studies provide a subjective insight into the performance of such applications. In this paper, we hypothesize that spatial coherence or the lack of it of the embedded virtual objects among users has a correlation to the QoE in MICE. We use Position Discrepancy (PD) to model this lack of spatial coherence in MICE. Based on that, we propose a Hierarchical Position Discrepancy Model (HPDM) that computes $\mathrm{PD}$ at multiple levels to derive the application/system-level PD as a measure of performance.

Experimental results on an example task in MICE show that HPDM can objectively quantify the application performance and has a correlation to the psychometric study-based QoE measurements. We envisage HPDM can provide more insight on the MICE application without the need for extensive user study.
\end{abstract}

\section{CCS CONCEPTS}

- Computer systems organization $\rightarrow$ Embedded systems; Redundancy; Robotics • Networks $\rightarrow$ Network reliability

\section{KEYWORDS}

Multi-sensorial; QoE; Modeling; Collaboration; Distributed; User experience; Metrics;
Permission to make digital or hard copies of all or part of this wo rk for personal or classroom use is granted without fee provided that copies are not made or distributed for profit or commercial advantage and that copies bear this notice and the full citation on the first page. Copyrights for components of this work owned by others than the author(s) must be honored. Abstracting with credit is permitted. To copy otherwise, or republish, to post on servers or to redistribute to lists, requires prior specific permission and/or a fee. Request permissions from Permissions@acm.org.

MMSys'17, June 20-23, 2017, Taipei, Taiwan

(C) 2017 Copyright is held by the owner/author(s). Publication rights licensed to ACM. ACM ISBN 978-1-4503-5002-0/17/06 ..\$15.00

http://dx.doi.org/10.1145/3083187.3084018

\section{INTRODUCTION}

Multi-sensorial, Immersive, Collaborative Environments (MICE) and applications are increasingly becoming popular in various applications such as tele-rehabilitation [1-3], teleoperation $[4,5]$, gaming $[6,7]$ with the availability of commodity hardware such as RGB-D cameras [8], haptics [9], inertial sensors [10], head mounted displays [11], etc. Collaboration among users in MICE typically involves Augmented/Mix ed/Virtual Reality (VR), through manipulation of synthetic 3D models (created using animation and modeling software such as Maya) and real-time captured (using RGB-D cameras) $3 \mathrm{D}$ objects. User manipulations of these objects are generally facilitated using haptics, vision-based gesture recognition devices, etc., apart from 'traditional' modes such as keyboard, mouse, or voice commands. MICE-based applications rely on distributed communication infrastructure. Hence, the network and operating system latencies $[12,13]$ in updating the changes in the objects' positions due to participating users' manipulations result in discrepancies in the spatial view or Position Discrepancy (PD) of the MICE-based applications at the participating users. As PD or lack of spatial coherence [14] among the participating users of MICE-based applications increase, QoE (Quality of Experience) on the task can degrade.

QoE in such MICE-based applications are, in general, measured using psychometric studies with Likert-type of scale evaluations. These evaluations provide QoE from an end-user point-of-view, but it is limited by its subjective observations based on (i) a user's bias in grading their experience (some are more critical than others); (ii) user's interest and concentration throughout the task; (iii) ease of use and comfort level of the interaction interfaces, (iv) task duration, (v) testing for different scenarios such as different network conditions, and (vi) importance of the application. Hence, in this work, we address two related questions:

1. Can we objectively quantify the application performance without the need for subjective user study?

2. Can we use the above objective performance measurement to understand the possible QoE? 
Our study shows that a majority of the previous works [15, 16] has followed either Likert-type scale or Mean Opinion Score (MOS) methods to evaluate QoE. Few studies [14,33] compute PD beforehand to observe the behavior of certain other application-oriented aspects (for instance, a virtual object's stiffness behavior for varying PDs was studied in [14]). The effect of PD on a 3D tele-immersion system was evaluated through a controlled user study experiment in [3, 18]. In [31, 32] well-known mathematical models were applied to map Quality of Service (QoS) parameters and user experience to compute QoE. Methodology to assess QoE through Bayesian analysis was proposed in [20]. The principal feature in all the abovementioned collaborative applications is the extensive use of visual or spatial modality. An approach/model that considers visual/spatial/positional discrepancy as a metric to assess QoE has not been explored so far.

Proposed Approach and Contribution of our work. We hypothesize that lack of spatial coherence as measured as PD, has a correlation to the QoE in MICE. In this context, we first devise an approach to model the PD of the embedded virtual objects visualized by the participating users, and second a method to estimate QoE. Based on that, we propose a Hierarchical Position Discrepancy Model (HPDM) that computes $\mathrm{PD}$ at multiple levels to derive the application/system-level PD (refer section 3.6) as a measure of performance. We then carry out user experience studies in an example MICE-based application and show that HPDM has a correlation to the psychometric study-based QoE.

The paper is org anized as follows. In Section 2 we provide the summary of the existing approaches to QoE and discuss their limitations. In Section 3 we present the notion of an object-level PD and describe how the proposed HPDM can compute objectlevel PD to system-level PD hierarchically. In section 4 we discuss on the example MICE-based task, its experimental setup, subjective and objective results, and the observed correlation between PD and QoE. Section 5 provides the limitations of proposed HPDM and directions for ongoing and future work.

\section{RELATED WORK}

We classify the existing approaches to QoE in the context of PD or spatial discrepancy into five major groups as follows.

The first category considers subjective assessments to investigate the network Quality of Service (QoS) impairments on QoE. Research work [15] investigated the effect of delay and jitter on haptic, audio and video transmission on a 2-user system. With the application-level QoS parameters such as operability of haptic device, sound output quality, video output quality and interstream synchronization quality, Mean Opinion Score (MOS) was obtained using multiple regression analysis. And with MOS, a subjective assessment has been performed to assess user experience or QoE. A similar approach using MOS was investigated to evaluate the effect of network delay on the operability of haptic interface and fairness between players for a 2-user, balloon bursting game for soft objects in [16]. With MOSbased QoE assessment, Ishibashi et al. [19] investigated the influence of network delay on olfaction aspect in a fruit harvesting game. Considering haptics as the only interaction modality, [7] discussed the observed MOS to network delay variations on a 2-user, air hockey game. MOS was evaluated by considering interactivity of mallet, the interactivity of puck and output quality of the game. Through a subjective user study, the temporal bounds of a "mulsemedia" (multi-sensorial media) application involving haptics, visual, olfactory, and air-flow interfaces were examined for better user experience was evaluated in [18]. Using a psychophysical approach [2] as a QoE measure, [3] analyzes view-disparity and inter-stream synchronization of a 2-user, 3D Tele-Immersive system. Eric et.al [21] evaluated the effectiveness of MACBETH (Management of Avatar Conflict by Employment of a Technique Hybrid) over the other existing techniques (rubber-band, and incremental-motion) through subjective user study on a 3D Maze game and observed the discrepancies between the seen and felt motion of user's hand.

In the second category, QoE has been measured by identifying application-specific metrics. The work mentioned in [6] suggests that the addition of haptics increased the QoE of a standalone, haptic-only, passive handwriting application. The features considered for evaluation are realism, usefulness, and haptic versus mouse comparison properties. In [18], the authors quantify QoE with application-defined metrics and figured out the threshold of those metrics through a controlled virtual environment study.

The third category studies proposed mathematical models to estimate QoE, by limiting either the number of interaction interfaces, or the nature of interaction itself. In [31], a linearweighted average mathematical model was applied to QoS parameters and user experience to compute QoE of a 2-user, haptic-only, 3D "balance ball" game. The follow-up work [32] compared different approaches for weight determination (even weight distribution, correlation-based weights, even weightscorrelation combination, linear reg ression analysis, and Principal Component Analysis (PCA)) approaches that can be applied to QoS parameters and user experience to determine QoE, the results demonstrated QoE with reference to haptics only limiting the number of modalities. A methodology to assess QoE through Bayesian analysis was proposed in [20] to a 2-user, haptic-audiovisual interactive system involving a passive task, the task was performed in one-way at a time, i.e., as the manipulator performs the task with haptics, the instructor observes the manipulator's actions through a haptic interface and vice versa - thereby restricting the nature of the task.

Research works $[14,33]$ of the fourth category investigate human capabilities to perceive the discrepancies between haptic and visual modalities. With five pre-computed values for spatial discrepancies, Lee et al. [14] explored the impact of visual-haptic discrepancies on human performance for precise targeting applications.

The fifth category studies [22-26] proposed time-delay compensation techniques such as wave variables, virtual coupling to reduce the consequence of spatial discrepancy. These works 


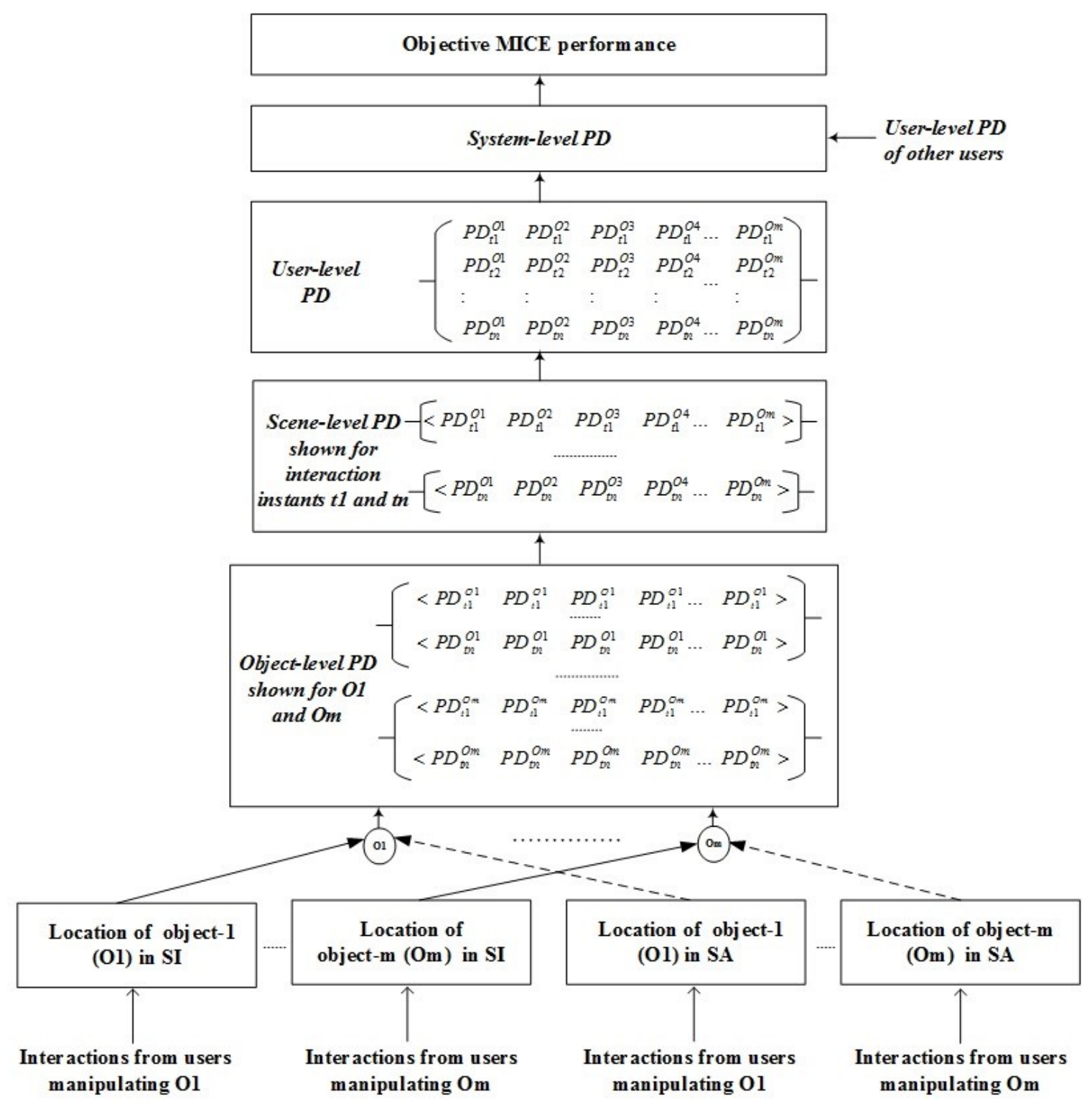

Figure 1: HPDM for a ' $k$ ' users, ' $m$ ' objects and ' $n$ ' interaction instants (scenes) MICE-based task.

evaluated the transparency among the participating users' in a distributed haptic-only interaction.

Our Observations.

1. Regardless of the system architecture, or the number of interaction interfaces, subjective assessments share common drawbacks - users' interest and skill level in using the system interfaces can easily impact QoE.

2. In [3] we observe that (i) the interaction object's mobility in the virtual space was confined, and (ii) the methodology to measure disparity was not addressed.
Whereas in our work we consider virtual objects mobility and we address a model that considers visual/spatial/positional discrepancy as a metric to assess QoE.

3. In [31, 32] well-known learning models were used to predict QoE by training on application-specific feat ures and the corresponding user study metrics, whereas, we propose a model to quantify QoE without a need for training/learning. 
4. In [14, 33], based on the application, discrepancies were pre-computed. The impact of discrepancy for varying virtual object stiffness on human perception were analyzed. These studies choose discrepancies within a certain range, but did not discuss the reason and the procedure for discrepancy pre-computation. In a similar way, [22-26] proposed various solutions to minimize spatial discrepancy, however, these studies did not discuss their approaches for spatial discrepancy computation.

\section{HPDM AND PD COMPUTATION}

In this section, we explain the proposed HPDM by considering a multi-sensorial, distributed system of ' $k$ ' users collaborating on a virtual task involving ' $m$ ' objects for ' $n$ ' interaction instants until task completion.

The foremost step in such a MICE-based application is to obtain the basic level of PD, which we denote as the object-level $\mathrm{PD}$ - i.e. the PD of a virtual object as observed by participating users. To accomplish this, we propose a log-based post-processing approach, in which, at each of the participating users, we log the virtual objects 3D locations until task completion in two scenarios: (i) a reference scenario, termed as Snapshot Ideal (SI), assuming any communication infrastructure with a minimal network delay, and (ii) a real scenario, termed as Snapshot Actual (SA) assuming any communication infrastructure with a network delay that is always greater than that of SI.

An object-level PD at a user for an interaction instant $t$ (where, $t \leq n$ ) is the misalignment in an object's 3D location in SA when compared with the same object's 3D location in SI. For instance, comparing with SI, an object has to be at a particular location $(\mathrm{X}, \mathrm{Y}, \mathrm{Z})$ but due to delay in receiving the interaction frames from other users', the object as in SA can be at a different location (A,B,C).

The proposed methodology of HPDM is shown in Fig. 1. In order to obtain object-level PD, virtual objects' 3D locations must be known. A virtual object's 3D location depends on the nature of a user's manipulation on a virtual object. In MICE-based application, we consider two types of object manipulations (i) directly-manipulated object, and (ii) non-manipulated object. If a user independently or by collaborating with other users manipulates an object then we refer to it as a directlymanipulated object (with respect to that user). If not, we refer to those objects as non-manipulated objects for that user (i.e., objects are not manipulated directly this user, but by someone else).

In the following sections, we describe the procedure to obtain virtual objects (directly-manipulated, non-manipulated) locations in SI and SA, and then we discuss the hierarchical PD computation approach of the proposed HPDM.

\subsection{Obtain 3D location of an object in Snapshot Ideal}

3.1.1 3D location of a directly-manipulated object. For users who collaboratively manipulate a shared virtual object, their directly- manipulated object is the same. Therefore, for collaborative manipulation, the directly-manipulated object's position depends not only on a user's local interaction but also on collaborators' interaction on the object. For users who manipulate the directlymanipulated objects with no collaboration, the 3D location of the objects depends only on a user's local interaction. Hence, at a user, for an interaction instant $t$, a directly-manipulated object's current/new 3D location is the summation of all of its interactions (local interaction and/or collaborator's interaction) to the object's previous $3 \mathrm{D}$ location as shown in (1),

$$
\left.O S_{S I}^{D M}\right|_{t=i}=\left.O S_{S I}^{D M}\right|_{t=i-1}+\sum\left(\text { int }_{i}\right)
$$

where SI is snaps hot ideal, OS is directly-manipulat ed object's 3D location, $D M$ stands for a directly-manipulated object and $t$ is an interaction instant, int is local and/or collaborator's interaction. As users collaborate, their interactions (int) on virtual objects are transmitted to other users in communication frames.

3.1.2 3D location of a non-manipulated object. At a user, a non-manipulated object's 3D location is the object's change in $3 \mathrm{D}$ location due to the interaction of the user's non-collaborator. Thus, a non-manipulated object's new 3D location is the summation of a user's non-collaborator's interaction to the corresponding non-manipulated object's previous 3D location,

$$
\left.O S_{S I}^{N M}\right|_{t=i}=\left.O S_{S I}^{N M}\right|_{t=i-1}+\sum\left(\text { int }_{i}\right)
$$

where $S I$ is snapshot ideal, $O S$ is non-manipulated object's 3D location, $N M$ stands for non-manipulated object and $t$ is an interaction instant, int is the remote interaction of a noncollaborator on a non-manipulated object.

\subsection{Obtain 3D location of an object in Snapshot Actual}

In $\mathrm{SA}$, we compute the 3D locations of directly-manipulated objects and non-manipulated objects as the same way as discussed in sections 3.1.1 and 3.1.2 using equations (1) and (2) respectively. We represent the directly-manipulated object's 3D location in SA as $O S_{S A}^{D M}$ and a non-manipulated object's 3D location in SA as $O S_{S A}^{N M}$, where $D M$ stands for directlymanipulated object, $N M$ stands for non-manipulated object, and $S A$ is snapshot actual.

\subsection{Object-level PD}

Knowing a virtual object's position at SI and SA, object-level PD can be computed. At each participating user, the object-level PD for an interaction instance $t$ is the misalignment in a virtual object's 3D location in SA when compared with the same virtual object's 3D location in SI. Therefore, object-level PD for a directly-manipulated object for an interaction instant, $t$ is,

$$
P D_{t}^{D M}=\operatorname{Diff}\left(O S_{S I}^{D M}, O S_{S A}^{D M}\right)
$$

where $O S_{S I}^{D M}$ is the $3 \mathrm{D}$ location of a directly-manipulated object in SI, and $O S_{S A}^{D M}$ is the $3 \mathrm{D}$ location of the corresponding directlymanipulated object in SA, and Diff is the Euclidean distance 
between the objects. Similarly, the object-level PD for a nonmanipulated object for an interaction instant, $t$ is,

$$
P D_{t}^{N M}=\operatorname{Diff}\left(O S_{S I}^{N M}, O S_{S A}^{N M}\right)
$$

Referring to Fig. 1, object-level PD for object-1 (O1) at interaction instant, $t 1$ is $P D_{t 1}^{O 1}$, object-level PD for object-2 (O2) at interaction instant, $t n$ is $P D_{t n}^{O 2}$, and so on.

Object-level PD can degrade MICE-based application performance, i.e., an increase in the object-level PD of a directlymanipulated object among the collaborating users can result in delayed responsiveness. For instance, consider a MICE-based task scenario in which user-1 (U1) and user-2 (U2) collaborate on object-1 (O1). Until U1 receives the delayed interaction frames on the shared object's interaction from U2, U1 can only achieve the local interaction response but not the responsiveness corresponding to U2's interaction on the shared object. As a result, both users might observe intermittent co-presence (the feeling that another user is also manipulating the same virtual object). Similarly, an increase in object-level PD of a nonmanipulated object can result in fluctuating visual rendering of the non-manipulated object - i.e., non-manipulated object's location can remain stationary for some time and then suddenly change.

\subsection{Scene-level PD}

A scene in a MICE-based application constitutes all of the virtual objects, irrespective of those being either a directly-manipulated object or a non-manipulated object. Therefore, with different object-level PDs, HPDM obtains a scene-level PD.

Scene-level PD is the discrepancies of all the virtual objects (directly-manipulated and non-manipulated) for any interaction instant $t$. Referring again to Fig. 1, scene-level PD for an interaction instant $t 1$ is $<P D_{t 1}^{O 1}, P D_{t 1}^{O 2}, P D_{t 1}^{O 3}, P D_{t 1}^{O 4} \ldots P D_{t 1}^{O m}>$, where, the virtual objects $(O 1, O 2 \ldots O m)$ can be either directlymanipulated or non-manipulated object.

As scene-level PD increase, a us er can view a different scene than that of other participating users. This might lead to a different interaction possibility, which in turn further increase the scene-level PD among the participating users.

\subsection{User-level PD}

Knowing scene-level PD for all interaction instants (or scenes) until task completion, HPDM can obtain user-level PD. Userlevel PD determines the amount of PD suffered by a user throughout all the scenes until task completion. With user-level $\mathrm{PD}$, a user would have viewed misaligned scenes than that of the other users.
As shown in Fig. 1, the user-level PD at U1 is the misalignment of all virtual objects $(\mathrm{O} 1, \mathrm{O} 2 \ldots \mathrm{Om})$ in all of the scenes $(t 1, t 2 \ldots t n)$ until task completion.

\subsection{System-level PD}

System-level PD is related to the MICE-based application performance over the entire task duration, considering all the users. By determining the PDs that all the users (U1, U2 ... Uk) perceive on all of the virtual objects $(O 1, O 2 \ldots O m)$ for all the scenes $(t 1, t 2 \ldots t n)$ until task completion, system-level PD determines which user could have perceived better performance when compared with the rest of the other users.

With this quantitative PD measurement proposal, we now objectively analyze the performance of the MICE-based task.



Figure 2: An example 3-user MICE system.

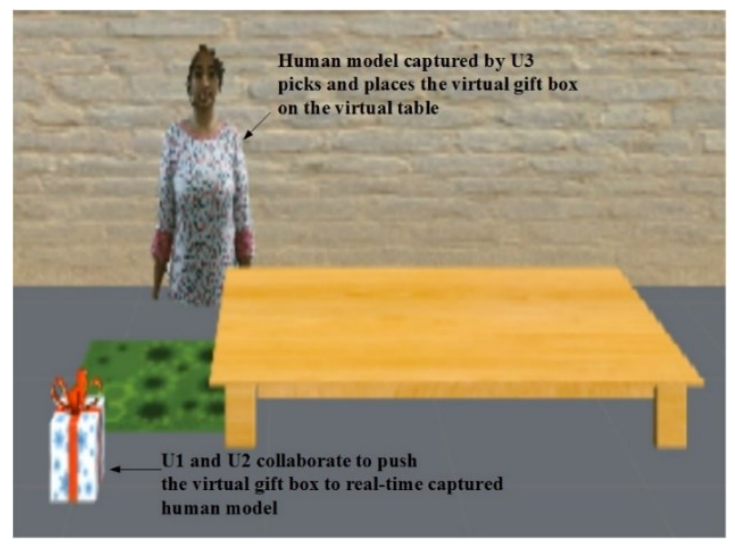

Figure 3: Example MICE based task with three user interactions on two virtual objects.

\section{EXPERIMENTAL SETUP AND RESULTS}

\subsection{Example MICE-based Application}


Table 1: User roles, virtual objects of interaction and interaction interfaces for 3-user, MICE-based task.

\begin{tabular}{lccc}
\hline & User-1 (U1) & User-2 (U2) & User-3 (U3) \\
\hline $\begin{array}{l}\text { User's directly-manipulated } \\
\text { object }\end{array}$ & Virtual gift box (O1) & Virtual gift box (O1) & $\begin{array}{l}\text { Real-time } \\
\text { model (O2) }\end{array}$ \\
\hline User's interaction interface & Haptics & Haptics & Kinect \\
\hline $\begin{array}{l}\text { Does this user collaborate with } \\
\text { any other user? }\end{array}$ & with U2 & with U1 & - \\
\hline User's non-manipulated object & $\begin{array}{c}\text { Real-time captured human } \\
\text { model (O2) }\end{array}$ & $\begin{array}{c}\text { Real-time captured human } \\
\text { model (O2) }\end{array}$ & Virtual gift box (O1) \\
\hline
\end{tabular}

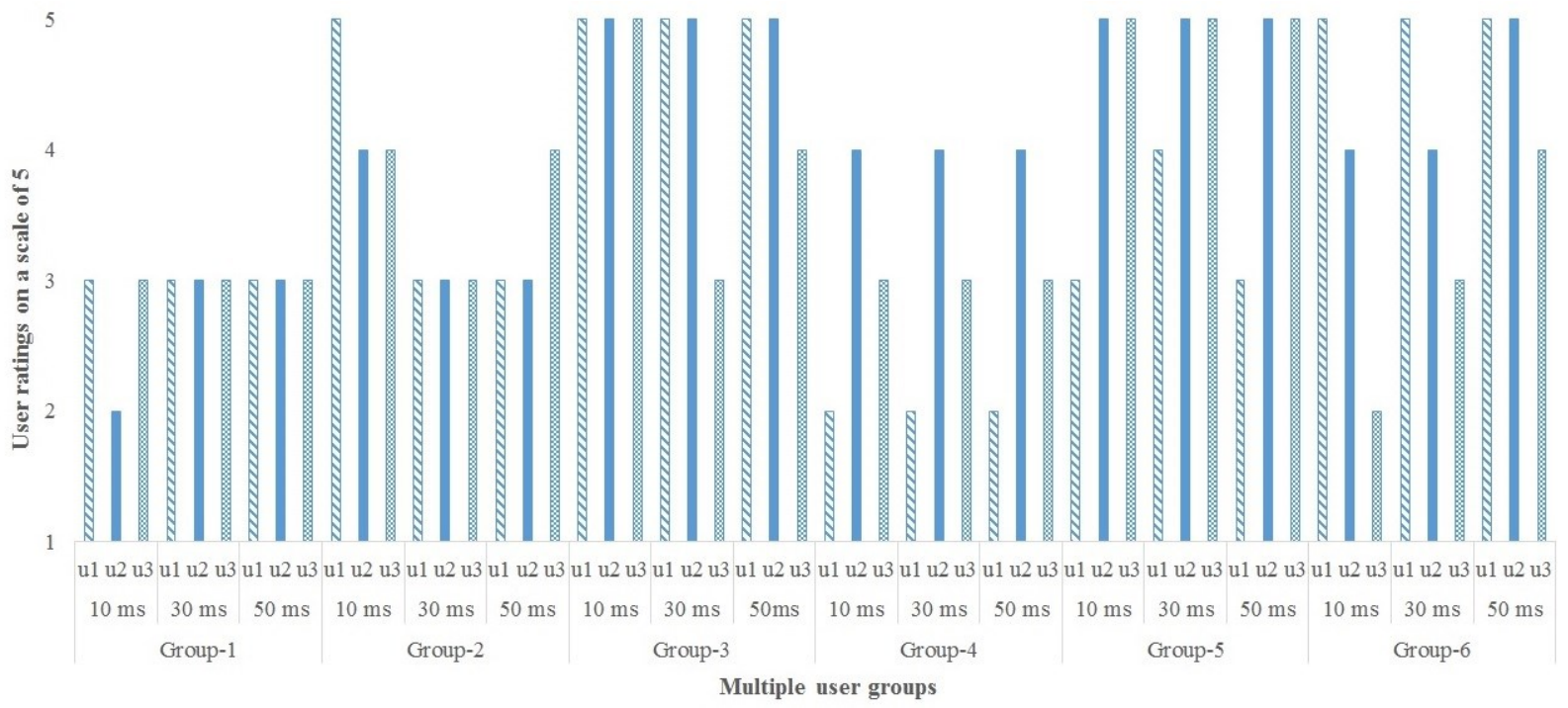

Figure 4: Perceived or user-level QoE (on a scale of 5) of the six user groups on the example 3-user, MICE-based task.

The effectiveness of the proposed HPDM was tested on a multisensorial, 3-user desktop-based distributed setup shown in Fig. 2. Time synchronization among the three desktop machines was achieved using NTP tool [27]. The MICE-based "Pick and Place" task shown in Fig. 3 was designed using Unity game eng ine [28]. The dimension of the MICE-based virtual environment is $160 \mathrm{x}$ $100 \times 100$ Virtual Units (VUs). In this MICE-based task, we consider two virtual objects (a synthetic 3D virtual gift box and a real-time captured 3D human model), the three participating users (U1, U2, and U3) can interact with one of the virtual objects using an interaction interface (haptics or Kinect). We design the task in such a way that each participating user manipulates a directly-manipulated object and depends on a non-manipulated object for the task completion. Referring to Fig.3, U1 and U2 collaborate to move virtual gift box (i.e., O1) with their respective haptic devices, and $\mathrm{U} 3$ captures a 3D human model (i.e., O2) in real-time, using Microsoft's Kinect. Thereby, U1 has a directly-manipulated object i.e., O1, and as U1 does not manipulate the real-time captured human model, it is a nonmanipulated object to U1. U1 and U2 move virtual gift box from its initial location to a final destination, where the real time captured 3D human model picks and places the virtual gift box on the virtual table. The task completes as and when the realtime captured human model places the virtual gift box on the virtual table. Table 1 shows the participating users' (U1, U2, and U3) role, their directly-manipulated object and non-manipulated object, and their interaction interface.

As we $\log$ the virtual objects 3D locations until task completion in SI and SA scenarios, we now explain on how we obtained thes e scenarios. Both, the SI and SA scenarios of MICE was performed on a 1 Gbps communication setup. For this communication setup, the maximum network delay that we observed in the reference or SI scenario was only $5 \mathrm{~ms}$. For SA scenario, a delay prone environment than that of SI is required, therefore, to achieve such environment, we induced additional network delay using Dummynet tool [29]. In our experiment, we have considered only three SA scenarios with network delay of $10 \mathrm{~ms}, 30 \mathrm{~ms}$, and $50 \mathrm{~ms}$ respectively. As the users performed the task in SI and SA scenarios, (i) user int eractions were collected, and object-level, scene-level, us er-level and system-level PD were obtained with the proposed HPDM, and (ii) perceived users' QoE was obtained through user study using a Likert-type 


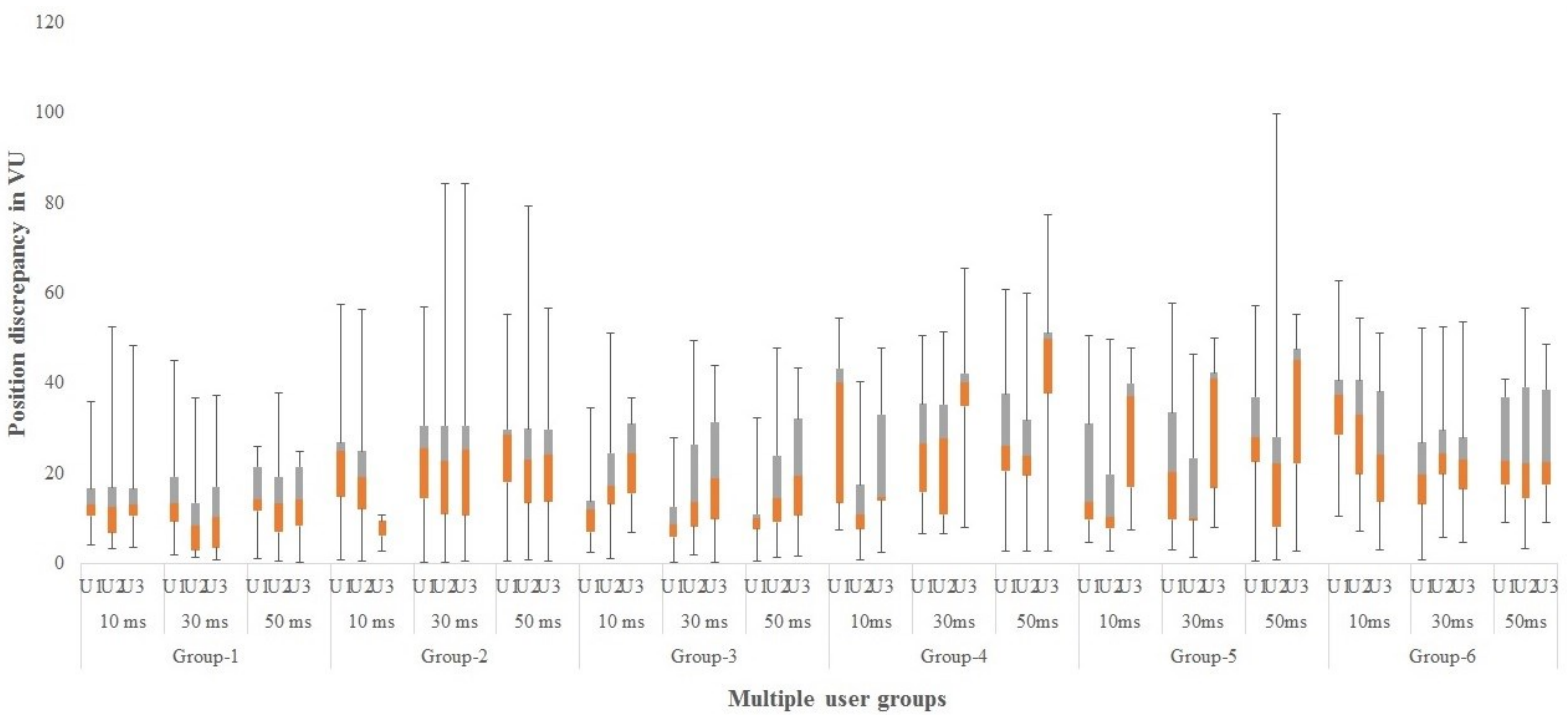

Figure 5: Scene-level PD for all the delay scenarios of the six user groups on the example 3-user, MICE-based task.
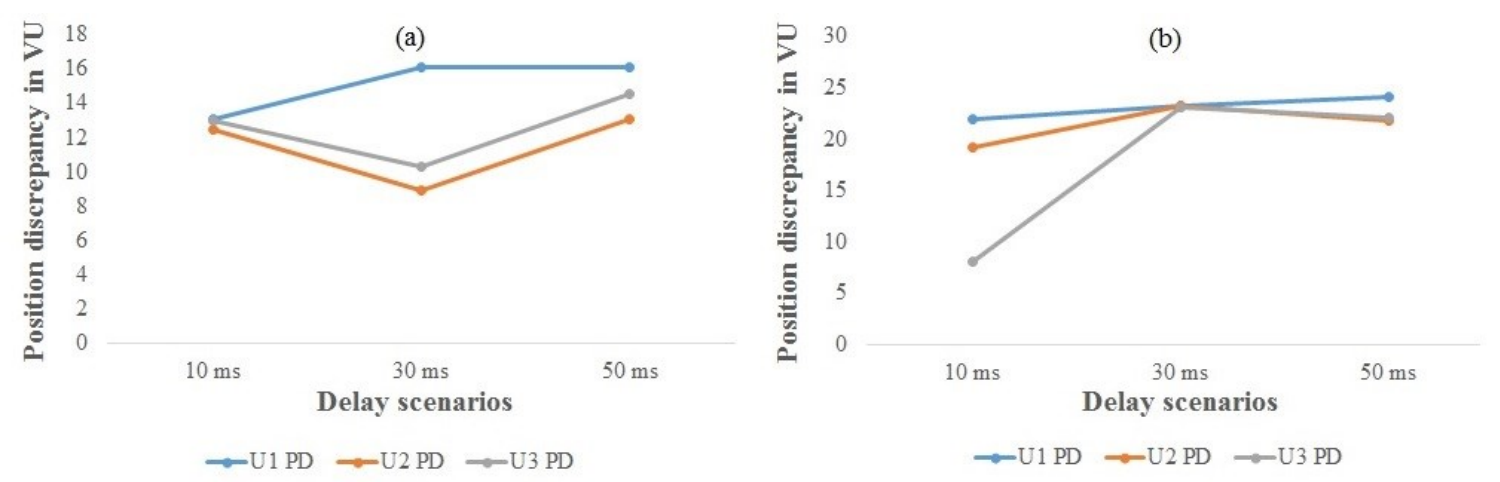

Figure 6: User-level PD of user group-1 (shown in (a)) and user group-2 (shown in (b)) on the example 3-user, MICE based task.
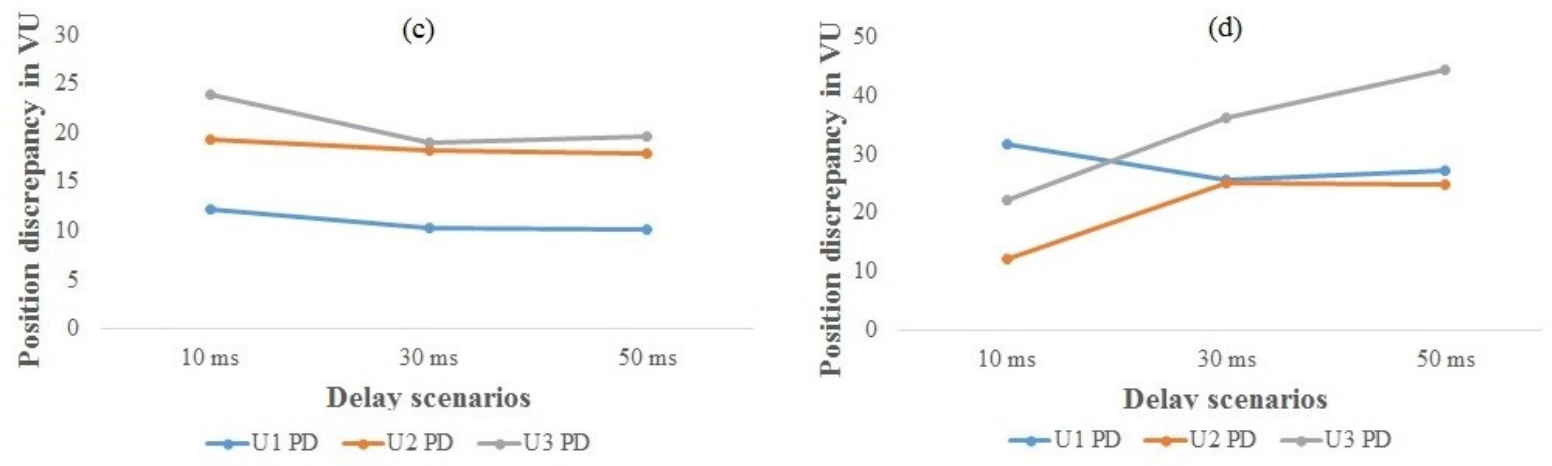

Figure 7: User-level PD of user group-3 (shown in (c)) and user group-4 (shown in (d)) on the example 3-user, MICE based task. 

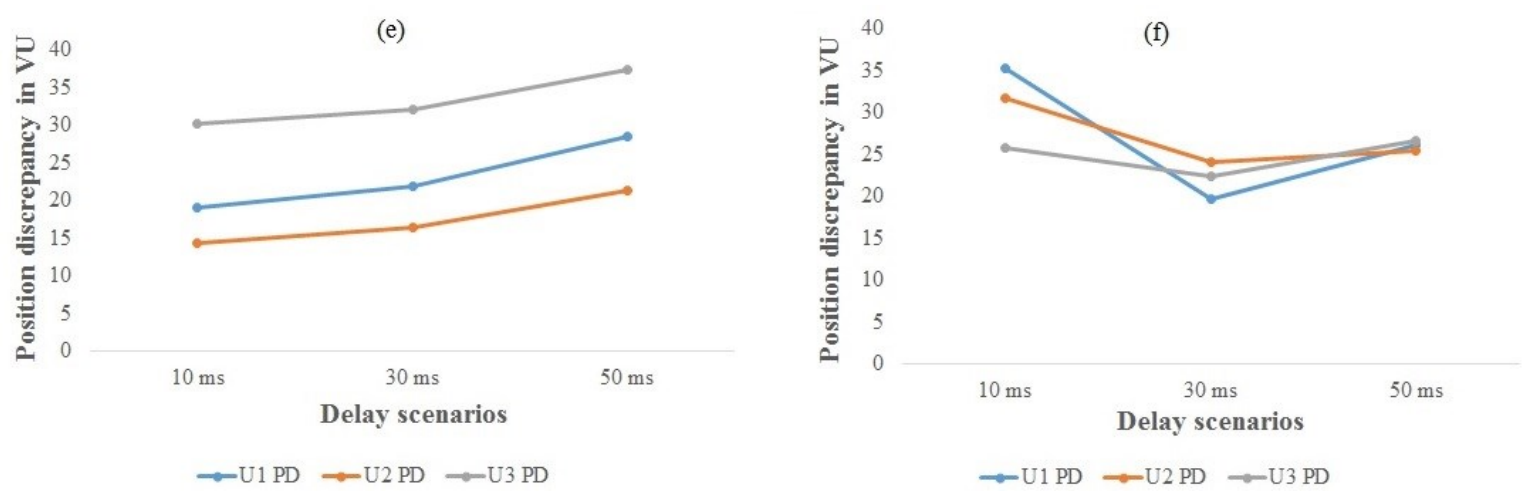

Figure 8: User-level PD of user group-5 (shown in (e)) and user group-6 (shown in (f)) on the example 3-user, MICE based task.

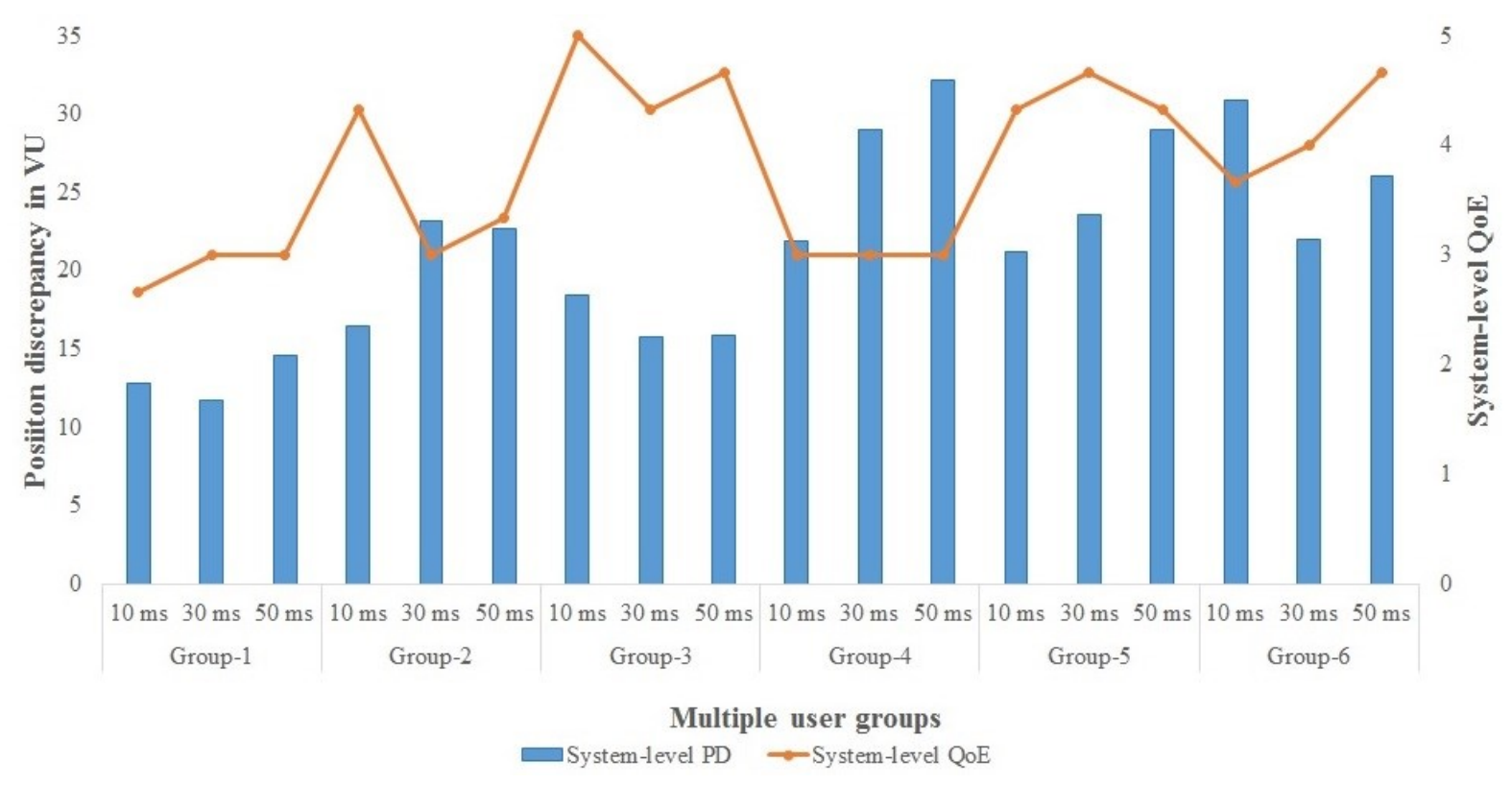

Figure 9: System-level PD of the six user groups on the example 3-user, MICE-based task.

questionnaire on a five-point scale (where a ' 1 ' is for poor performance and a ' 5 ' is for best performance).

In the following sections, we discuss the results as obtained from user study and HPDM.

\subsection{Subjective Results}

A total of 18 users (8 Novice, 4 Intermediate, and 6 Experts), both Male and Female in the age group of 24 to 35 years participated in the user study. As the example MICE-based task is a 3-user system, in a user study, three users were considered as a user- group, and six such groups of user evaluations were performed. In a user group, three users performed the MICE-based task for SI and SA scenarios. As users finished the task, their evaluations on the task were obtained through the Likert-type questionnaire. The length of a user group evaluation typically varied from 30 to 45 minutes, this includes device setup and calibration, performing the MICE-based task for SI and three SA scenarios $(10 \mathrm{~ms}, 30 \mathrm{~ms}$, and $50 \mathrm{~ms}$ ) and evaluating the questionnaires for SI and three SA scenarios. A similar process was followed in the rest other user groups. 


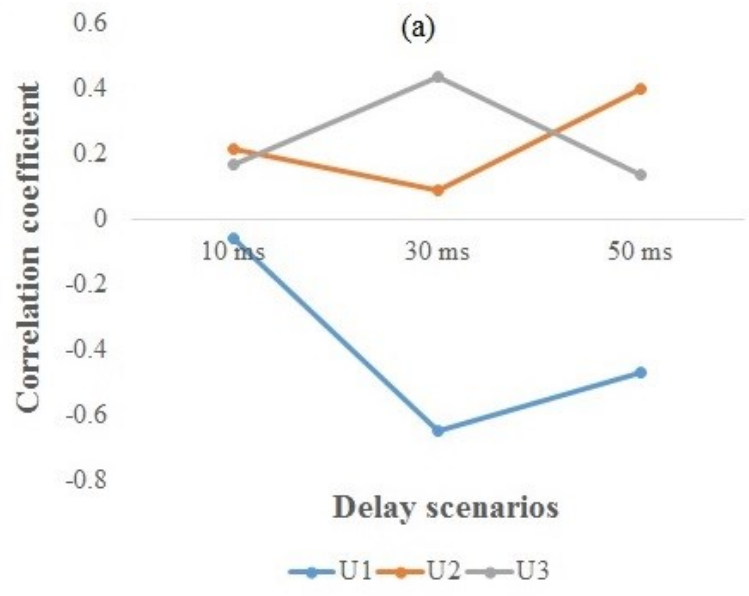

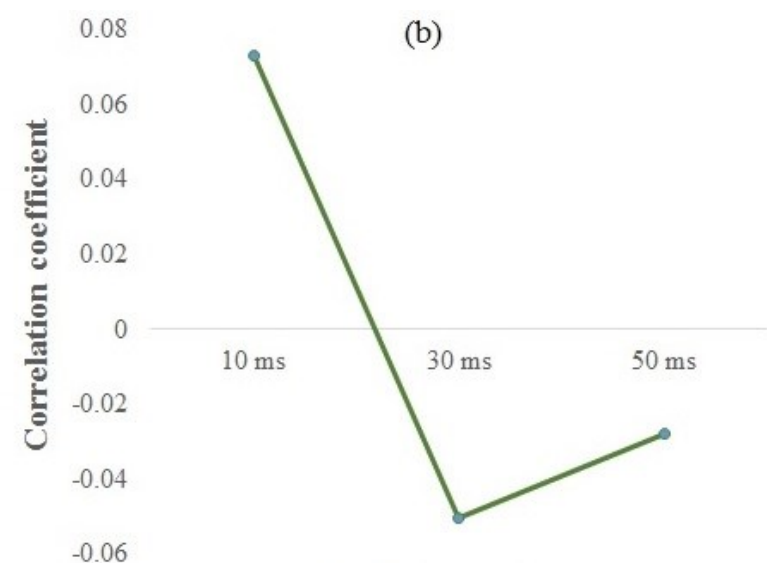

Delay scenarios

Figure 10: Correlation between user-level PD to user-level QoE is shown in (a), and correlation between system-level PD to system-level QoE is shown in (b).
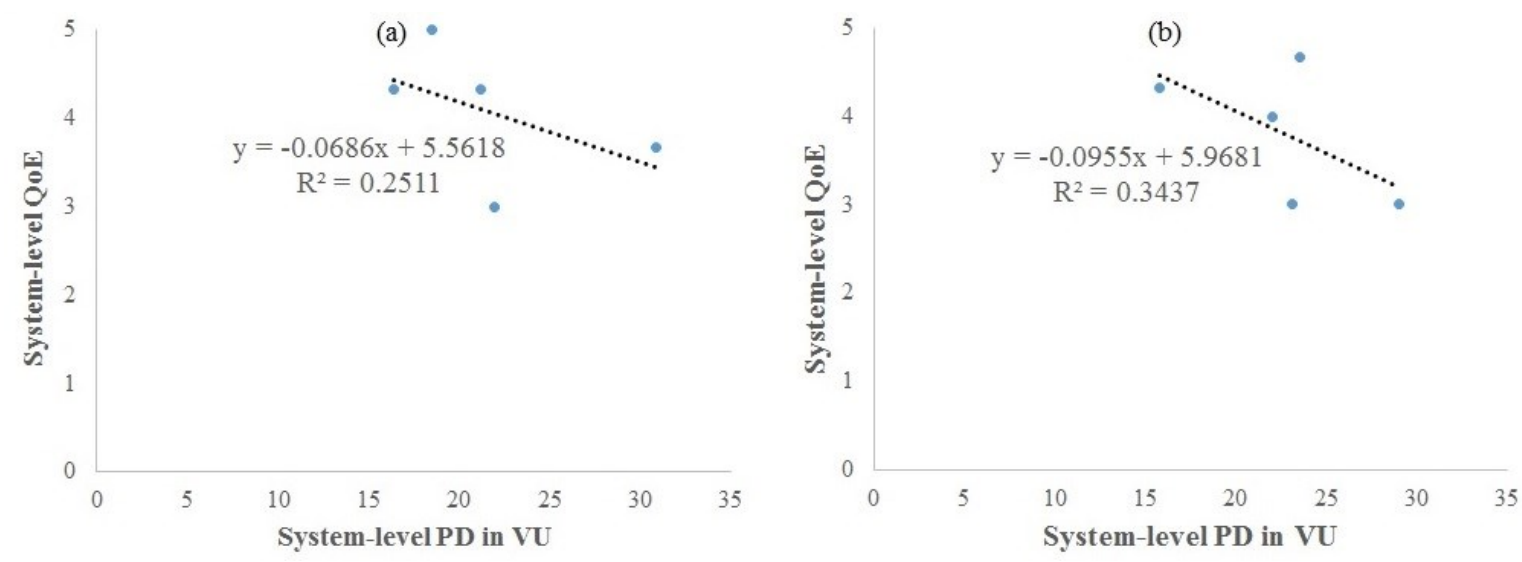

Figure 11: Estimated system-level QoE for $10 \mathrm{~ms}$ and estimated system-level QoE for $30 \mathrm{~ms}$ are shown in (a) and (b), respectively.

Users' evaluations for the performance aspect of the MICEbased task as perceived or user-level QoE rating for all the user groups are shown in Fig. 4. First, for a delay scenario of a g roup, the perceived or user-level QoE for the MICE-based task across the users of that group is rated quite differently, for instance, in the $10 \mathrm{~ms}$ delay scenario of group-1, U2 rating is different than that of U1 and U3. It is quite reasonable as each user might had a different opinion on the task and system performance and challenging to decide on QoE especially as users' rating for the same scenario varies. Second, it can be observed that there is a significant difference in the user rating of a delay scenario across all the groups. For example, the user rating as U1 for $10 \mathrm{~ms}$ delay scenario across all the six user groups show significant variation. Finally, we observe that the impact of various network delay conditions on MICE-based task had no significant impact on the perceived or user-level QoE in certain groups, for instance, user- level QoE of user group-4 remain the same across all the delay scenarios. With these observations, it is understandable that user QoE as perceived by users is limited by its subjective evaluations. Therefore, relying on MICE-based system performance through user QoE can cause more chaos than good.

\subsection{HPDM Results}

Here we discuss our observations on various levels of PD as obtained from HPDM, and then we quantify the MICE-based task performance with HPDM's final outcome (i.e., system-level PD measurement).

4.3.1 Quantify MICE performance through HPDM. In this section, we address the research question: Can we objectively quantify the MICE application performance without the need for a subjective user study? For each of the six user groups that evaluated the MICE-based task, with their directly-manipulated 
and non-manipulated object-level PDs, scene-level PDs for each of the six user groups are obtained. Scene-level PDs of the participating users (U1, U2, and U3) for all the six user groups for $10 \mathrm{~ms}, 30 \mathrm{~ms}$, and $50 \mathrm{~ms}$ delay scenarios are shown in Fig. 5. At any user, scene-level PD provides the amount of PD suffered by all the virtual objects in the virtual environment for each of the interaction instants until task completion. By observing the PD spread, minimum PD, and maximum $\mathrm{PD}$ of each of the participating users (U1, U2, and U3) of the six user groups as shown in Fig. 5, the results clarifies that each of the participating users must have suffered with relatively different PDs until task completion. It is this substantial difference of PD that contribute to the difference in user-level PD among U1, U2, and U3. The average of the scene-level PD at a participating user is considered as the participating user's user-level PD. User-level $\mathrm{PD}$ of the three participating users (U1, U2, and U3) of user group-1 to user group- 6 for $10 \mathrm{~ms}, 30 \mathrm{~ms}$ and $50 \mathrm{~ms}$ delay scenarios are shown in Fig. 6 through Fig. 8. User-level PD helps in disting uishing the MICE-based task experience among the participating users. User-level PD results for user-group-1 (shown in Fig. 6 (a)) signifies that U2 and U3 would have experienced a much better MICE-based task experience than U1.

To obtain the overall MICE-based task performance, we examine the system-level PD as obtained by HPDM. A systemlevel PD of a user group for a delay scenario $10 \mathrm{~ms}$ is the average of the user-level PDs of U1, U2 and U3 of that user group as observed for the $10 \mathrm{~ms}$ delay scenario. A comparison of systemlevel PD of all user groups is shown in Fig. 9. Of all the six user groups, users of group-1 suffered with less PD (PD of 12.82 VU for $10 \mathrm{~ms}$, PD of $11.75 \mathrm{VU}$ for $30 \mathrm{~ms}$, and PD of $14.57 \mathrm{VU}$ for 50 $\mathrm{ms})$. This indicates that the participating users of user group-1 must have experienced better MICE-task performance when compared with the rest other user groups (i.e., user group-2 to user group-6), supporting the claim that with HPDM MICEbased task performance can be obtained objectively.

Referring again to system-level PD results shown in Fig. 9, we observe that system-level PD of certain us er groups at $10 \mathrm{~ms}$ are more than the system-level PD at any higher delay scenario of the same user group. Three instances of this case are observed in our study on system-level PD, those are in user group-1, user group-3 and user group-6. These results indicate that apart from network delay, other factors have been contributed to MICEbased task performance: (i) slow and/or fast user interactions during SI, (ii) slow and/or fast user interactions during SA, and (iii) user's response to the impact of network delay and thereby slowing down their objects movements. These can be some of the reasons for system-level PD variation of $10 \mathrm{~ms}$ delay scenario of certain groups to be more than that of any higher delay scenario in the same user group.

4.3.2 HPDM to obtain possible QoE. In this section, we address the research question: Can we use the above objective performance measurement to understand the possible QoE?

To inspect the possibility to model QoE with objective measurement of HPDM, we consider two cases: (i) user-level QoE, and (ii) system-level QoE. For a user group, user-level QoE (shown in Fig.4) is the perceived QoE of each user. Similarly, for a user group, the system-level QoE (line graph in Fig. 9) is the average perceived QoE of all the users. For instance, the systemlevel QoE of a user group for delay scenario $10 \mathrm{~ms}$ is the average of the perceived users' QoE of U1, U2, and U3 for $10 \mathrm{~ms}$ of that user group. Now, to obtain possible QoE:

1. Identify the correlation between user-level QoE and user-level PD.

2. Identify the correlation between system-level QoE and system-level PD.

3. Based on the observations on the correlation coefficients of system-level QoE and system-level PD, we propose a modeling approach to estimate systemlevel QoE.

With the user study data collection and HPDM results, we look for an association between QoE and PD. A correlation analysis using Pearson method [30] on user-level PD and perceived user QoE (i.e., user-level QoE) ratings from user study shows the inverse association between user-level PD and userlevel QoE. In Fig.10 (a), of all the users that performed the task as U1 in all the six user groups there exists an inverse relationship between their user-level PD and perceived user QoE ratings. Though U2 follows a similar trend as U1, the correlation is a very minimal direct relationship among user-level PD and userlevel QoE at all the users that performed the MICE-based task assuming the role of U2. The variation in the perceived or userlevel QoE ratings between the users playing the roles of U1 and $\mathrm{U} 2$, though they collaborate in the MICE-based task to move the same object, shows the subjective nature of QoE measurement.

In a similar way, we perform a correlation analysis using Pearson method on system-level PD and system-level QoE

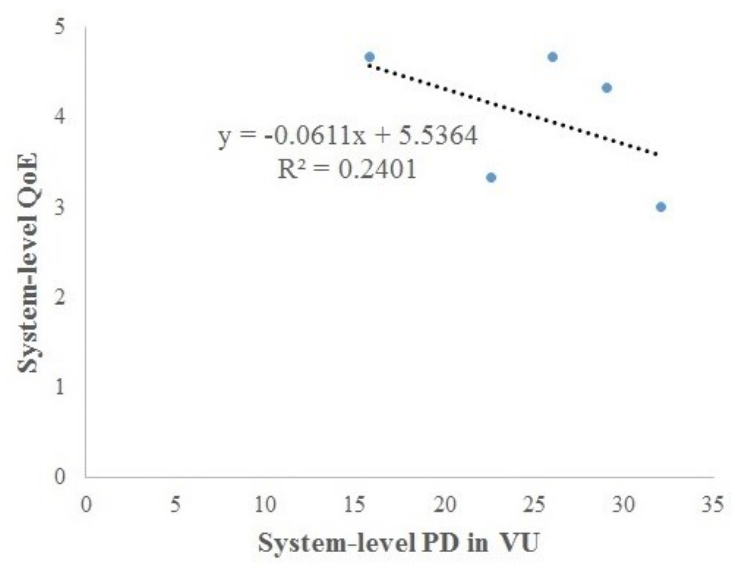

Figure 12: Estimated system-level QoE for $50 \mathrm{~ms}$ network delay.

(shown as line graph in Fig. 9) ratings. We observe the same trend of inverse relationship between system-level QoE and system-level PD which is shown in Fig. 10 (b). Correlation analysis of system-level PD to system-level QoE shows a minimal inverse correlation of -0.05 and -0.02 for $30 \mathrm{~ms}$ and 50 $\mathrm{ms}$ scenario respectively, and a minimal linear correlation of 0.073 for $10 \mathrm{~ms}$. 
The results of both the correlation analysis (user-level PD and user-level QoE in Fig. 10 (a) and system-level PD and systemlevel QoE shown in Fig. 10 (b)) provide the evidence that the linear relationship between HPDM and the perceived QoE can be used to model possible QoE. Therefore, with linear regression approach, we model system-level QoE as a function of systemlevel PD. The results of linear regression to system-level PD and system-level QoE for $10 \mathrm{~ms}$, and $30 \mathrm{~ms}$ delay scenarios are shown in Fig. 11 and the same for 50 ms delay scenario is shown in Fig. 12.

Regression statistics (R-square value that determine how well the system-level PD and system-level QoE data fit to linear regression model, slope and intercept) for each of the delay scenarios are provided in Table 2. The reason for low R-square value at all the delay scenarios is primarily due to the variations of the perceived or user-level QoE ratings (shown in Fig. 4), that depends on factors such as users' behavior, us er skill level, users' concentration, etc.

Table 2: Regression Statistics

\begin{tabular}{lcc}
\hline Network & \multicolumn{2}{c}{ Regression Statistics Summary } \\
\cline { 2 - 3 } Delay & & \\
\hline \multirow{2}{*}{$10 \mathrm{~ms}$} & Intercept & 5.56177 \\
& Slope & -0.06859 \\
& R Square & 0.2510 \\
& & \\
$30 \mathrm{~ms}$ & Intercept & 5.9680 \\
& Slope & -0.0955 \\
& R Square & 0.3436 \\
& & \\
& Intercept & 5.5364 \\
& Slope & -0.0611 \\
& R Square & 0.2400 \\
\hline
\end{tabular}

Summary. The correlation between user-level PD and userlevel QoE is rather minimal, presumably because of individual preferences in giving QoE ratings (shown in Fig. 4). However, there is a clear correlation between system-level PD and systemlevel QoE. This is reflected in Fig. 11 and 12 showing that a high system-level PD means a low predicted system-level QoE and vice versa.

\subsection{How to use HPDM to any other MICE- based task?}

The performance of an application quantified using HPDM (as discussed in section 4.3.1) is useful to understand the QoE of other MICE-based tasks, though the results discussed in section 4.3.2 are specific to the scenario described in Section 4. For other MICE-based tasks, our suggestion is to carry out minimal user QoE study using psychometric evaluations under few delay scenarios and using proposed approach in section 4.3.2 to obtain system-level QoE for few different delay scenarios. Then, HPDM can track system performance during regular use of the MICEbased tasks. Now, the obtained system-level QoE can be correlated with the obtained system-level PD (similar to Fig. 11 and 12). This system-level PD to system-level QoE correlation, can then be used to understand system-level QoE without doing psychometric evaluations every time. For instance, if a group of users carrying out the MICE-based task experiences a systemlevel PD of $x$ units, then we can estimate that the corresponding system-level QoE could be $y$. Hence, using the proposed HPDM to estimate system-level QoE can significantly reduce the timeconsuming psychometric evaluation process in obtaining user QoE.

\section{CONCLUSIONS}

In this paper, we proposed a model-based approach that can measure positional discrepancy among the participating users in a MICE-based task to objectively quantify the task performance and also estimate QoE.

We demonstrated HPDM capabilities on an example 3-user, MICE-based task with a psychometric evaluation of user QoE involving 18 users. Our results indicate that there is an inverse association between QoE and PD at both us er-level and systemlevel. System-level PD to system-level QoE results confirmed a negative correlation between QoE and PD. A linear regression model is then shown to be useful for estimating the possible QoE.

The benefits of HPDM are three-fold: (i) It provides the PD suffered by all the virtual objects at various levels throughout the task completion, (ii) each level of PD measurement feeds to the next higher level of PD measurement, making the approach computation flexible, and (iii) with the objective system-level PD measurement, the performance of any MICE-based application can be figured out and QoE can be assessed.

However, in this work, user behavioral aspects (such as skill level, sustained interest throughout the task scenario, fatigue level, comfort level on the system interfaces, etc.,) and its influence on the perceived or user-level QoE were not addressed. Another limitation is that the proposed HPDM model cannot classify intentional non-cooperation and/or unintentional non-cooperation (due to less experience with the MICE interfaces) interactions of the participating users. Both these interactions are treated the same, contributing to $\mathrm{PD}$ at different levels. We would investigate how to distinguish between these interactions and improve the proposed HPDM in our future work. 


\section{A USER STUDY QUESTIONNAIRE}

A Likert-type questionnaire with a five-point scale (where a ' 1 ' is for poor performance and a ' 5 ' is for best performance) was followed to evaluate MICE-based task performance. The following questions were evaluated by participating users' after performing the MICE-based task in SI and three SA $(10 \mathrm{~ms}, 30$ $\mathrm{ms}$, and $50 \mathrm{~ms}$ ) scenarios.

1. Rate your task performing experience in this multiuser scenario.

2. Were you able to complete your part of the task?

3. How helpful was multi-user interactions to achieve the task?

4. How frequent did you feel other users' presence while performing the task?

5. How was virtual gift box visual rendering quality?

6. How would you rate virtual gift box movement?

7. How was 3D human model's visual rendering quality?

8. Rate your observation on virtual gift box movement due to collision (if any) with other objects.

9. Relative to your object of manipulation, how will you rate movements of other virtual objects?

10. Rate your overall experience.

\section{ACKNOWLEDGMENTS}

This material is based upon work supported by the National Science Foundation (NSF) under Grant No. 1012975 and US Army Research Office (ARO) STIR (Short Term Innovative Research) Grant 67369-CS. Any opinions, findings, and conclusions or recommendations expressed in this material are those of the author(s) and do not necessarily reflect the views of the NSF and ARO.

\section{REFERENCES}

[1] W. Wu. Color-plus-depth level-of-detail in 3d tele-immersive video: a psychophysical approach. In Proceedings of the 19th ACM international conference on Multimedia. ACM, New York, NY, USA, 2011, MM '11, pp.1322.

[2] K. Venkatraman, S. Raghu raman and B. Prabhakaran. 2013. Describing a view alignment framework in 3D Tele-immersion systems. International Conference on Multimedia and Expo Workshops (ICMEW). IEEE San Jose, CA, pp. 1-4.

[3] K. Venkatraman, S. Raghu raman, Y. Tian, B. Prabhakaran, K. Nah rstedt and T. Annaswamy. 2014. Quantifying and Improving User Quality of Experience in Immersive Tele-Rehabilitation. International Symposium on Multimedia (ISM). IEEE. Taichung, pp. 207-214.

[4] A. Tatematsu, Y. Ishibashi, N. Fukushima and S. Sugawara. 2011. QoE assessment in tele-operation with 3D video and hapt ic media. International Conference on Multimedia and Expo. IEEE. Barcelona, pp. 1-6.

[5] Yushing Cheung and J. S. Chung. 2009. Cooperative control of a multi-arm system using semi-autonomous tele-manipulation and adaptive impedance. International Conference on Advanced Robotics, Munich, pp. 1-7.

[6] A. Hamam, N. D. Geo rgan as and A. E. Saddik. 2012. Effect of haptics on the Quality of Experience. International Symposium on Haptic Audio-Visual Environments and Games (HAVE). IEEE. Phoenix, AZ, pp. 1-6.

[7] Y. Kusunose, Y. Ishibashi, N. Fukushima and S. Sugaw ara. 2010. QoE assessment in networked air hockey game with haptic media. 9th Annual Workshop on Network and Systems Support for Games (NetGames). Taipei, pp. 1-2.

[8] https://developer.microsoft.com/en-us/windows/kinect

[9] www.geomagic.com

[10] https://www.xsens.com/tags/inertial-sensors/

[11] https://www.sony.co.uk/pro/article/medical-3d-head-mounted-display
[12] M. Glencross, C. Jay, J. Feasel, L. Kohli, M. Whitton and R. Hubbold. 2007. Effective Cooperative Haptic Interaction over the Internet. Virtual Reality Conference. IEEE. Charlotte, NC, 2007, pp. 115-122.

[13] Kian Meng Yap, A. Marshall and Wai Yu. 2007. Supporting haptic collaboration across networked peers with real time force feedback in distributed virtual environments. International Conference on Telecommunications and Malaysia International Conference on Communications. IEEE. Penang, pp. 494-499.

[14] C. G. Lee, I. Oakley, E. S. K im and J. Ryu. 2016. Impact of V isual-Hapt ic Spatial Discrepancy on Targeting Performance. In Transactions on Systems, Man, and Cybernetics: Systems. IEEE. vol. 46, no. 8, pp. 1098-1108

[15] A. Tatematsu, Y. Ishibashi, N. Fukushima and S. Su gawara. 2010. QoE assessment in haptic media, sound and video transmission: Influences of network latency. International Workshop Technical Committee on Communications Quality and Re liability (CQR 2010). IEEE. V ancouver, BC, pp. 1-6.

[16] M. Sithu, Y. Ishibashi, P. Huang and N. Fukushima. 2015. QoE assessment of operability and fairness for soft objects in networked real-time game with haptic sense. 21st Asia-Pacific Conference on Com munications (APCC). Kyoto, pp. 570-574.

[17] Z. Yuan, G. Ghine a and G. M. Muntean. 2014. Quality of experience study for multiple sensorial media delivery. International Wireless Communications and Mobile Computing Conference (IWCMC). Nicosia, pp. 1142-1146.

[18] Z. Yuan, T. Bi, G. M. Muntean and G. Ghinea. 2015. Perceived Synchronization of Mulsemed ia Services. In Transactions on Multimedia. IEEE vol. 17, no. 7, pp. 957-966.

[19] Y. Ishibashi, S. Hoshino, Qi Zeng, N. Fukushima and S. Su gawara. 2012. QoE assessment of fairness between players in networked game with olfaction. 11th Annual Workshop on, Network and Systems Support for Games (NetGames). Venice, pp. 1-2.

[20] Tasaka. 2016. Bayes ian structural equation modeling of multidimensional QoE in haptic-audiovisual interactive communications. International Conference on Communications (ICC). IEEE. Kuala Lumpur, pp. 1-6.

[21] E. Burns, S. Razzaque, M. C. Whitton and F. P. Brooks. 2007. MACBETH: The avatar which I see before me and its movement toward my hand. Virtual Reality Conference. IEEE Charlotte, NC, pp. 295-296.

[22] G. Sankaranarayanan and B. Hannafo rd. 2008. Experiment al comparison of internet haptic collaboration with time-delay compensation techniques. International Conference on Robotics and Automation. IEEE. Pasadena, CA, pp. 206-211.

[23] S. Lee and J. Kim. 2009. Delay Compensation Scheme for Transparency over Haptic-Based Networked Virtual Environments. International Conference on Complex, Intelligent and Software Intensive Systems. Fukuoka, pp. 961-966.

[24] Z. Li and D. Constantinescu. 2009. Networked haptic cooperation among multip le users via virtual object coordination to a veraged position of peer copies. International Workshop on Haptic Audio visual Environments and Games. IEEE. Lecco, pp. 170-175.

[25] Y. Ye, Y. J. Pan and Y. Gupta. 2009. Time domain passivity control of teleoperat ion systems with random asymmet ric time delays. Proceedings of the 48th Conference on Decision and Control (CDC) held joint ly with 2009 28th Chinese Control Conference. IEEE. Shanghai, pp. 7533-7538.

[26] S. Shimizu, T. Matsunaga and K. Ohnishi. 2015. Method of position erro compensation in consideration of symmetric structure for bilateral control system under time delay. 41st Annual Conference of the IEEE Industrial Electronics Society. IECON. Yokohama, pp. 004633-004638.

[27] http://www.ietf.org/rfc/rfc5905.txt

[28] Unity3d.com

[29] http:info.iet.unipi.it luigidummynet.

[30] G Hall. 2015. Pearson's correlation coefficient. http://www.hep.ph.ic.ac.uk/ hallg/UG_2015/Pearsons.pdf.

[31] A. Hamam and A. El Saddik. 2012. Evaluating the Quality of Experience of haptic-based applications through mathematical modeling. Intemational Workshop on Haptic Audio Visual Environments and Games (HAVE). IEEE. Munich, pp. 56-61.

[32] A. Hamam and A. E. Saddik. Toward a Mathematical Model for Quality of Experience Evaluation of Haptic Applications. In Transactions on Instrumentation and Measurement. IEEE. vol. 62, no. 12, pp. 3315-3322, Dec. 2013.

[33] M. Scandola, L. Gasperotti, M. Vicentini and P. Fiorini. 2012. The role of visual-haptic discrepancy in virtual reality environments. Haptics Symposium (HAPTICS). IEEE. Vancouver, BC, 2012, pp. 289-295. 